ISSN: $1130-3743$

\title{
LA HIPÓTESIS DE LA PEDAGOGÍA POSTMODERNA. EDUCACIÓN, VERDAD Y RELATIVISMO
}

\author{
The hypothesis of postmodern pedagogy. Education, truth \\ and relativism
}

\section{L’hypothèse de la pédagogie postmoderne. Éducation, vérité et relativisme}

\author{
Xavier LAUDO CASTILLO \\ Universidad de Barcelona. Facultad de Pedagogía. Departamento de Teoría e \\ Historia de la Educación. C/ Passeig Vall d'Hebron, 171. Ed. Llevant. 08035 \\ Barcelona.Correo-e: xlaudo@ub.edu
}

Fecha de recepción: marzo de 2011

Fecha de aceptación definitiva: julio de 2011

Biblid [(1130-3743) 23, 2-2011, 45-68]

\section{RESUMEN}

Este estudio retoma el debate sobre la pedagogía postmoderna con una doble finalidad: Por un lado, para contribuir a un orden conceptual más claro y coherente sobre lo pedagógico en relación a la Postmodernidad. Por otro, para, sobre este orden teórico, despejar el camino de lo que podrían ser o están siendo teorías y prácticas educativas constituyentes de una pedagogía, de suyo, postmoderna. La investigación se ha llevado a cabo a través de la hermenéutica de textos filosóficos y pedagógicos. En primer lugar, se presenta el binomio moderno/postmoderno en su nivel epistemológico y se clarifica su relación con el relativismo. Segundamente, se discute y defiende la posibilidad y la existencia de una pedagogía de signo postmoderno, teniendo especialmente en cuenta su vertiente normativa. En tercer lugar, se exponen los argumentos que, desde un punto de vista hermenéutico y pragmatista, el relativismo y sus matices ofrecen como línea pedagógica. Se ensaya en esta parte una integración del postfundacionalismo 
y otros postulados postmodernos en el pensamiento educativo, y se apuntan algunas líneas a desarrollar en el futuro. Finalmente, se concluye con una síntesis de los resultados y sus implicaciones para la pedagogía y la teoría de la educación.

Palabras clave: Postmodernidad, epistemología, hermenéutica, pragmatismo, valores sociales, teoría de la educación, filosofía de la educación.

\section{SUMMARY}

This article proposes to recover the debate on the postmodern pedagogy with a double aim. On the one hand, contribute to clarify and make more coherent the conceptual order on pedagogy in relation to Postmodernism. On the other, clearing the way for the real possibilities of what might be or are being, educational theories and practices constitutive of a postmodern pedagogy. The research was conducted through the hermeneutic of philosophical and pedagogical texts. First, the postmodern is presented in the epistemological level and clarifying his relation to different types of relativism. Second, we discuss and defend the possibility and the existence of a postmodern pedagogy taking into a special account the question of the normativity. Thirdly, we develop the arguments that, from a hermeneutic and pragmatist view, relativism offered as educational trend. In this part we offer an integration of postfoundationalism and other postmodern principles in educational thinking and suggests some lines to develop in the future. Finally, we conclude with a synthesis of the outcomes and their implications for pedagogy and educational theory.

Key words: Postmodernism, epistemology, hermeneutics, pragmatism, social values, educational theories, educational philosophy.

\section{SOMMAIRE}

Cet article revient sur le débat sur la pédagogie postmoderne avec un double objectif. D'une part, aider à clarifier et rendre plus cohérente l'ordre conceptuel sur la pédagogie par rapport au postmodernisme. De l'autre, ouvrant la voie à des possibilités réelles de ce qui pourrait être ou sont en cours les théories et les pratiques éducatives qui constituent une pédagogie postmoderne. La recherche a été menée grâce à l'herméneutique des textes philosophiques et les méthodes d'enseignement. Premièrement, nous présentons le postmoderne sur le plan philosophique en relation avec différents types de relativisme. Deuxièmement, nous discutons et défendons la possibilité et l'existence d'une pédagogie postmoderne en tenant compte en particulier de son aspect normatif. Troisièmement, nous exposons les arguments à partir d'un point de vue herméneutique et pragmatiste que le relativisme nous offre comme tendance pédagogique. Dans cette partie, nous proposons une intégration de postfondationnalisme et d'autres principes de la pensée postmoderne en éducation, et nous suggérons quelques lignes à développer à l'avenir. Enfin nous terminons avec un résumé des conclusions et de leurs implications pour la pédagogie et la théorie de l'éducation.

Mots clés: Postmodernité, epistémologie, herméneutique, pragmatisme, valeurs sociales, théories de l'éducation, philosophie de l'éducation. 
LA HIPÓTESIS DE LA PEDAGOGÍA POSTMODERNA. EDUCACIÓN, VERDAD Y RELATIVISMO

\section{INTRODUCCIÓN}

En la primavera de 1995 se publicó un número de Educational Theory donde se afirmaba lo siguiente: "Un creciente número de educadores, encontrándose en problemas por los valores y asunciones modernistas, están girándose hacia el postmodernismo en busca de ayuda para entender y articular sus preocupaciones" (Child, Williams, Birch y Boody, 1995, 167). Desde entonces, y algo antes, en pedagogía se viene haciendo uso, a veces abuso, ya sea sustantiva o adjetivamente, de términos como "Postmodernidad" o "postmoderno". En muchas ocasiones se esquiva su significado profundo y, como si se tratara de un burladero conceptual, se apela a la oposición a la vacuidad postmoderna como parapeto donde cualquier teoría en apuros, falta de una legitimación y representatividad perdidas que ansía recuperar, puede refugiarse. Es por esto que juzgamos pertinente retomar este debate, que en España ya cuenta los veinticinco años, y entre los que se distinguen episodios preclaros como los que supusieron los trabajos de Colom (1984), Gervilla (1993), Fullat (2002a) y Ayuste y Trilla (2005).

Antes de entrar en la exposición de los contenidos debemos hacer una aclaración. Y es que habrá advertido el lector que hay una cierta ambivalencia en la primera parte del título del artículo. Cabe, pues, empezar por resolver esa duda inicial. ¿Cómo interpretar el genitivo del enunciado? Si le diéramos al sintagma el sentido de un genitivo objetivo aludiríamos a la hipótesis que los pedagogos postmodernos asumen como cierta para llevar a cabo su pedagogía postmoderna. Lo que implicaría la asunción, más o menos consciente o reflexionada, de que es posible educar en y desde valores postmodernos. En cambio, si leemos el genitivo como subjetivo, nos estamos refiriendo a la hipótesis que los pedagogos no convencidos de la viabilidad o deseabilidad de una pedagogía postmoderna tienen ante sí y se plantean esclarecer. El presente trabajo toma en cuenta ambas direcciones de investigación. Al último sentido se dedica especialmente el apartado tercero: la pedagogía postmoderna como hipótesis para intentar dilucidar de qué modo la podemos considerar pedagogía en sentido estricto, y qué tipo de planteamientos educativos se podrían inscribir en ella. Sobre el primer sentido, la hipótesis defendida y convertida en tesis por los argumentos del pensamiento postmoderno, se trata más concretamente en el apartado cuarto.

Como última advertencia antes de comenzar, querríamos consignar que un estudio completo de la condición social postmoderna debería tener en cuenta al menos dos niveles distintos: por un lado, el de la epistemología y, por otro, el de su ontología social (Best, 1998, 312; Terrén, 1997, 123). El primero, más bien un paradigma que un periodo de la historia, se refiere al estatuto de la realidad, su accesibilidad y la posibilidad de formular predicados con aspiraciones de verdad. Este nivel será en el que caracterizaremos nuestra presentación de la Postmodernidad y a través del cual articularemos su traducción pedagógica y discutiremos la hipótesis ya mencionada. El segundo nivel es el que trata de la esencia o forma característica de constituirse y desarrollarse lo social y las relaciones entre actores en la sociedad 
postmoderna. Aquí sí que nos encontramos ante un conjunto de nuevos fenómenos sociales susceptibles de considerarse como una fase o época histórica distinta de las anteriores, algunas de cuyas características serían: la flexibilización de las identidades, la reorganización social del tiempo en el cortoplacismo, la individualización, el neonomadismo, la licuescencia del compromiso, etc. (Laudo, 2008). No obstante, aunque queremos dejar aquí constancia de la importancia pedagógica de este análisis sociológico, no lo desarrollaremos en este artículo, pues el propósito principal es contribuir a desentrañar y esclarecer la cuestión filosófico-educativa de la hipótesis pedagógica postmoderna.

\section{El RELATIVISMO O LA POSTMODERNIDAD EPISTEMOLÓGICA}

A menudo se ha impugnado el término "Postmodernidad" por el vacío de contenido positivo que supone todo "post". Sin tener en cuenta, quizá, que "Modernidad" es también un concepto relativo puesto que se es moderno en relación a una etapa anterior de la historia considerada precisamente como antigua. Sea como fuere, y en coherencia a esa denuncia, lo postmoderno es casi siempre detractado por lo débil de sus certezas y lo efímero de sus proyectos. Sin embargo, no es menos cierto que el considerar esos adjetivos como indeseables en sí mismos responde al discurso de una tradición particular, histórica y contingente, la occidental, y no a ninguna suerte de criterio independiente que pueda mostrar cuál es la tradición o los juicios correctos. Y es esta última afirmación la que empieza ya a ser un lugar común en Occidente ${ }^{1}$.

En aras de la claridad expositiva concretaremos primero qué significamos con el concepto "Modernidad", entendiendo que, tanto éste como su derivado post, son conceptos abiertos que señalan un conjunto de formas simbólicas ${ }^{2}$. Los grandes vectores y relatos a través de los que se vertebra la Modernidad son los siguientes: la creencia en un horizonte de progreso y perfección del futuro; la identificación del triunfo de la razón con la linealidad y la meta de la historia; la educación de la sociedad como una misión de la élite cultural para lograr la emancipación individual y colectiva;

1. Un ejemplo bien representativo de ese pensamiento débil normativo lo encontraríamos en la filosofía taoísta, de cuyo libro de cabecera, el Tao Te Ching, se cuentan por decenas las distintas ediciones realizadas sólo en España en las dos últimas décadas. Uno de sus pasajes más conocidos (LXXVIII) reza: "Nada en el mundo es más blando y débil que el agua; mas no hay nada como el agua para erosionar lo duro y lo fuerte". Probablemente este progresivo reconocimiento postmoderno de la no universalidad de la tradición propia haya constituido un terreno abonado para la recepción de modelos orientales como el que acabamos de citar. Además, pueden reconocerse numerosas concomitancias entre el pensamiento postmoderno y la pedagogía del Tao. $C f$. LAUDO, 2011.

2. Usaremos el presente como tiempo verbal para referirnos a la Modernidad y no el pretérito, como podría esperarse, puesto que consideramos que los presupuestos y el imaginario modernos conviven con los postmodernos en régimen de coexistencia y no de exclusividad. 
y una visión representacional del mundo según la cual hay una realidad objetiva a la que puede accederse mediante la aplicación de un método (Terrén, 1997). Una "metafísica realista" basada en un mundo que es uno y singular, que existe independientemente del observador y que está ahí fuera esperando ser descubierto (Parker, 1997, 115). Seguramente, la clave de bóveda del proyecto moderno consista en la aspiración al conocimiento objetivo de la realidad, lo que Panikkar llamó "el mito" del conocimiento objetivo de la realidad, donde mito es aquello en lo que se cree sin creer que se cree, lo que se toma como natural (Panikkar, 2007), mientras que en el debate postmoderno lo que se discute es precisamente si hay realidad, irrealidad o multiplicidad de realidades (Lyon, 1994). En la concepción del conocimiento asociada a la Modernidad éste se concibe en permanente cambio. Un cambio que, a su vez, se entiende como mecanismo de ajuste progresivo a la realidad. De modo que, aunque las teorías se reconozcan esquemas conceptuales aproximativos, predomina la creencia de que "el conocimiento siempre es como movimiento de aproximación gradual a la verdad absoluta" (Cabrera, 2005, 47). En otras palabras, desde el punto de vista moderno, asumiendo la noción platónica de conocimiento, éste sólo puede serlo "cuando su contenido es verdadero. Conocer es, pues, pensar la verdad" (Zubiri, 1926, 289). Pero, en general, la crítica postmoderna no niega ni la realidad, por un lado, ni que ésta sea el referente de la ciencia, por otro. Aunque existe cierta discusión sobre si "lo real es precisamente aquello independiente de lo que nosotros o una mente cualquiera pueda pensar" (Nubiola, 2001, 55), las ciencias sociales no pueden más que tratar de abordarlo por los métodos existentes en las distintas disciplinas. No hay objeción nihilista en esto, ni en lo que ya Husserl concluyó reduciendo fenomenológicamente y partiendo de Descartes de que la conciencia sólo es condición de posibilidad de todo posible sentido de los objetos que conceptualizamos, no de su existencia real (Husserl, 1987). La crítica postmoderna a la que nos referimos no confunde (contra lo que dice David Carr, 2005) el sentido factual del conocimiento con el explicativo. Lo que esa crítica hace es, precisamente, "distinguir entre realidad y objetividad, entre los hechos realmente existentes y la forma en que son captados y explicados" (Cabrera, 2005, 39), para poner en cuestión la accesibilidad a la verdad entendida de forma objetivamente absoluta, ya que, se sostiene, la investigación objetiva, aunque perfectamente posible y muchas veces real, proporciona sólo algunas de las muchas formas de describir y comprender (Rorty, 1995, 326-327). Lo que se niega, pues, es que la verdad pueda ser el saldo resultante de los aciertos y los fracasos de una comunidad de científicos donde unos sirven a otros para llegar cada vez más lejos hasta -en palabras de Peirce-, "realizar el asalto de la ciudadela de la verdad trepando sobre los cadáveres de las teorías y experiencias fallidas" (Peirce, citado en Nubiola, 2001).

Pasando ya a abordar la significación de la Postmodernidad, ésta es, según la definición canónica de Lyotard, la crisis basada en la incredulidad respecto a los grandes relatos como legitimadores del saber (Lyotard, 1994, 9-10). Ya se trate de un relato de emancipación de la humanidad como de uno de especulación y 
conocimiento científico de la realidad (ibid., 63, 73). El mismo Lyotard refirió el cambio en los términos siguientes: "Las sociedades denominadas modernas fundan sus discursos de verdad y justicia sobre grandes relatos históricos y también científicos, unos y otros se colocan en la línea de una impresionante odisea progresista. Pues bien: en las sociedades postmodernas, en las cuales vivimos, lo que no se encuentra es precisamente legitimación de lo verdadero y lo justo... Ya nadie cree en salvaciones globales" (Lyotard y Descamps, 1984, 151-152). Este descrédito de los grandes relatos puede situarse históricamente en la segunda mitad del siglo XX (entre la II postguerra mundial, el mayo del 68 y la caída del muro de Berlín en 1989) y filosóficamente a partir de la obra de Nietzsche en relación a la afirmación de la muerte de Dios, por haber mostrado que la ordenación racional de la realidad a partir de una imagen metafísica era sólo un "mito tranquilizador" (Fullat, 2005, 415). Quedaron así, extraviadas para la mayoría, las referencias sólidas que antaño indicaron el camino. De modo que, según la afirmación nietzscheana, proféticamente adelantada a finales del siglo XIX, en el tiempo que iba a venir (el nuestro) "habrá[n] cambiado de sitio los mojones", y "los propios mojones volarán por el aire" (Nietzsche, 1999, 273).

Si nos centramos en el tema del relativismo, cabe destacar que la hermenéutica filosófica que atraviesa el siglo xx -y su obra fundamental, Verdad y método, considerada como una de las bases de la Postmodernidad (Fullat, 2002b, 127) - ya plantea la relatividad como algo insalvable. En palabras del propio Gadamer, esta filosofía, que debe entenderse "no como una posición absoluta, sino como un camino de experiencia, consiste en que no se da un principio superior al de abrirse al diálogo. [...] Mantener una posición propia parece inadmisible. ¿No significa ese diálogo interminable, en su última radicalidad, un relativismo total?» (Gadamer, 1994, 399). El asunto del relativismo va a ser piedra angular de toda la filosofía postmoderna. No obstante, hay que insistir en que la tesis relativista está lejos de ser un escepticismo en el sentido de que nada pueda conocerse. Lo que dice esa tesis es que nada de lo que puede conocerse puede tener rango de absolutidad o de universalización racionalmente admisible. De ahí que se consideren ese tipo de aspiraciones como características de la Modernidad y configuradoras del sujeto moderno como "un heredero de la ansiedad cartesiana, alguien que no puede aceptar la ambigüedad, la falta de claridad y certeza, ni la diversidad de opiniones a menos que concluyan en un acuerdo racional" (Bárcena, 2005, 56).

En síntesis, y antes de pasar a sopesar la hipótesis de la pedagogía postmoderna, lo que hemos querido subrayar hasta aquí es: lo que caracteriza a la Postmodernidad es una tesis relativista, relacionada con la constatación de lo imposible del conocimiento objetivo absoluto en cualquier orden, ya sea de verdad, belleza o bondad ${ }^{3}$.

3. Es cierto que puede resultar algo inconveniente identificar postmodernidad con relativismo. Por un lado, cuando se entiende el relativismo en su concepto llamado "ingenuo" o "irrestricto", 
Se trata del cada vez mayor primado de la interpretación y de lo contextual para considerar cualquier predicado como verdadero. En la feliz expresión de Jacobo Muñoz: la "relativización de lo humano al contexto" (Muñoz, 1997, 17-27). A su vez, es importante no confundir, como suele hacerse, el relativismo con la afirmación de la invalidez de nuestro modo de conocimiento o de nuestros códigos morales. Lo que el relativista afirma es la inadmisibilidad racional de universalizar normas, creencias o prácticas que, sin dejar de ser válidas, lo son en ámbitos y contextos concretos, cosa que le diferencia del escéptico o del cínico (Arenas, Muñoz y Perona, 1997, 9-10). En la sección cuarta del artículo retomaremos este asunto. Pero antes vamos a abordar la pregunta sobre si puede haber una pedagogía postmoderna.

\section{LA POSIBILIDAD PEDAGÓGICA POSTMODERNA}

Ya hemos esbozado que la Postmodernidad significa la "no aceptación de la existencia de realidad alguna que se presente como absoluta, autónoma y suficiente" (Fullat, 2002a, 341). Una vez instalada la crítica a esos fundamentos, se sigue de ellos que "la relación pedagógica ya no puede ampararse en las metanarrativas y universales característicos de la cultura pedagógica de la Modernidad" (Terrén, 1997, 121). De hecho, parece asentado que todo esto ha ido conformando un "desdibujamiento normativo" que ha llevado a hacer cada vez más "difícil señalar qué está bien o mal" y ha provocado que, cada vez más, aquellas legislaciones, profesores, textos o teorías que marcan fuertemente unas orientaciones de valor frente a otras nos parezcan adoctrinadores y los juzguemos como peligrosos (Gil, 2008 , 28). Tiene sentido, pues, que la pedagogía, dada la importancia y la sustancialidad de los cambios ya presentados, deba replantearse. Sin embargo, y más allá de la discusión sobre la conveniencia de un planteo en una u otra dirección, la pedagogía postmoderna ha sido negada no sólo en cuanto a su existencia, sino también en cuanto a su posibilidad. Para discutir esa doble negación tomaremos como base un valioso y leído artículo de Ayuste y Trilla (2005) en el que desarrollaron esta idea.

\footnotetext{
éste resulta autorrefutador. Además, en ese extremo de radicalidad resulta igualmente insostenible porque, como suele decir Rorty, la afirmación de que una tradición sea tan racional o tan moral como cualquier otra sólo podría ser sostenida por un dios. Por otro lado, también se puede aducir que la clave del postmodernismo es la imposibilidad del conocimiento objetivo, de lo que el relativismo sería sólo una de sus consecuencias. Pero girar el argumento puede también dar una explicación histórica congruente, es decir, que sea el advenimiento del relativismo lo que hace constatar y compartir la imposibilidad del conocimiento objetivo. Agradezco al historiador Victorio Heredero su disponibilidad e interés por discutir éste y otros puntos, lo que me ayudó a mejorar los contenidos y la claridad de este artículo.
} 
Asumamos, de momento, que "sin intención normativa no hay pedagogía" (Trilla, 2005, 293). Y que, además, para que algo sea considerado pedagogía debe cumplir tres condiciones: referirse a la educación en una perspectiva global; que haya una voluntad de coherencia interna; y que incluya como parte esencial contenidos de carácter normativo (Ayuste y Trilla, 2005, 220-221). Establecido esto, veamos cuál fue el cuestionamiento de la pedagogía postmoderna. Se articuló sobre dos negaciones: "No hay pedagogías relevantes elaboradas desde el pensamiento postmoderno", y eso se debe a que "no puede haberlas" (ibid., 231). Y esto porque "la mayoría de propuestas o resultan triviales o ya se hallan contenidas (y mejor desarrolladas) en las pedagogías modernas, o acaban siendo contradictorias con los propios presupuestos postmodernos, o no resistirían la misma crítica que dirigen a las pedagogías modernas, o son simplemente retóricas e inescrutables" (ibid., 237). En síntesis, las objeciones presentadas fueron básicamente tres: 1) su inconcreción retórica, 2) su "no postmodernidad", puesto que ya estaría contenida y realizada en una pedagogía moderna y 3) su contradictoriedad o falta de resistencia de la propia crítica.

La primera negación (no hay pedagogías postmodernas) se podría cuestionar desde, al menos, dos flancos: a) apelando a la imposibilidad empírica de sostenerla ad infinitum, b) impugnando el uso de alguno de los términos del enunciado. Respecto al primero, y menos importante: sea cual fuere la definición de Postmodernidad que manejáramos, siempre podría argüirse que, a la hora de afirmar la inexistencia de pedagogías postmodernas, lo que ocurriera fuera que no las viéramos. Es decir, siempre podría haber maestros o pedagogos por ahí, desconocidos o no publicistas, con un conjunto de disposiciones educativas armadas en una base experiencial o teórico-práctica, con perspectiva de globalidad, coherencia y normatividad, todo ello alimentado por un discurso postmoderno. Sin embargo, creemos que el problema radica en lo que apuntábamos desde el segundo flanco: el uso adjetivo de lo postmoderno, que es lo que puede llevar a negar su posibilidad pedagógica de iure, y luego, por razonamiento lógico, también su existencia de facto. Por lo tanto, hay que contemplar las dos negaciones a la vez, puesto que la primera (no hay pedagogías postmodernas) tiene que depender de la segunda (no puede haberlas) ${ }^{4}$.

Para fundamentar las tres objeciones planteadas se analizó un texto de Jacques Derrida sobre la universidad y el panfleto político de Jean-Claude Michéa La escuela de la ignorancia (que, por cierto, y no es baladí, lleva por subtítulo "y sus condiciones modernas") (Michéa, 2002). En referencia al texto de Derrida sobre la universidad es palpable que no se encuentra en él ni un atisbo de planteamiento

4. Y no al revés, puesto que, aunque nos es técnicamente imposible demostrar que no hay pedagogías postmodernas en ninguna parte, sí que podríamos evidenciar su imposibilidad ideal, por ejemplo, del mismo modo que podemos evidenciar la imposibilidad de pensar y realizar -aunque no de enunciar- un decaedro regular. 
global normativo y, por tanto, por lo que hasta aquí hemos asumido, ni de pedagogía. Sobre Michéa, nosotros no lo consideramos precisamente un postmoderno ni un adalid de la Postmodernidad, sino más bien todo lo contrario. Su obra, un alegato contra "los actuales progresos de la ignorancia", "el declive constante de la inteligencia crítica", una escuela que no favorece la "tradición revolucionaria" ni aprender "a partir de qué condiciones la rebelión contra el mundo se convierte en una necesidad moral" y mina "la capacidad de resistencia intelectual" (ibid., 14-15), nos parece más bien propia de la Modernidad, y de una Modernidad muy sólida. Y sobre lo que no hay duda es que, aunque habla de educación, no hay en él pedagogía, ni normativa ni basada en una aspiración de coherencia, y es, ciertamente, sólo "una crítica epatante pero sin compromiso plausible alguno" (Ayuste y Trilla, 2005, 239). Sobre la primera objeción (la inconcreción retórica), es evidente que no hay pedagogía en ninguno de estos dos trabajos, como tampoco la habrá en otros referidos a las nuevas tecnologías, la multiculturalidad o el derrumbe de los grandes relatos, siempre que sean únicamente descriptivos y críticos, puesto que ya hemos compartido que, en efecto, no puede haber pedagogía en un discurso que se quede en ese nivel.

Vayamos a la segunda objeción. Se insiste en que «los enunciados generales que se presentan con una apariencia de gran actualidad en realidad son muy poco originales" (ibid., 240-241). Esta enmienda también nos parece rechazable, precisamente por el problema de definición que avanzábamos, porque se basa en una caracterización muy sesgada de lo que sería una pedagogía postmoderna. Y es que como enunciados pedagógicos postmodernos se enumeran: la reivindicación del sentimiento, el carpe diem, el valor de las diferencias, el hedonismo, el cuerpo, la educación estética, la cultura popular y el relativismo (ibid., 240). Lo que ocurre es que, exceptuando solamente el último enunciado de la retahíla, éstos no son características esenciales del pensamiento postmoderno, sino contenidos contingentes que pueden ser, y efectivamente en muchos discursos postmodernos lo son, compartidos tanto con el imaginario social moderno (Taylor, 2002) como con las pedagogías modernas. Lo que a nuestro juicio puede distinguir y caracterizar una pedagogía acorde con los supuestos postmodernos -tal como los hemos caracterizado en el segundo apartado- es el tipo de fundamento desde y para el que se educa, y el rango de relatividad/universalidad que se otorga a sus contenidos. Por lo tanto, hay que concluir que no hay contradicción en que una pedagogía postmoderna pueda usar métodos y/o prescribir contenidos ya ensayados o inventados en la Modernidad y/o por pedagogías modernas.

La tercera objeción es, en efecto -aunque sus formuladores no desarrollaron específicamente este argumento-, la que más discusión admite. Y es que, desde un pensamiento estrictamente pedagógico, el único elemento que cabría ser planteado como nudo gordiano de la hipótesis pedagógica postmoderna reside en la característica de la normatividad. Entendida ésta como la parte de una pedagogía en la que se propone la validez de un modo y no la de otros sobre cómo se deben hacer las cosas, parecería en un primer momento contradecirse con el principio relativista 
postmoderno: el de que toda afirmación sólo es verdadera en relación a algún tipo de supuesto histórico y no de forma absoluta. El postmoderno diría, por ejemplo, "hay demasiada escuela en la escuela, excesiva norma", aseverando que el mayor problema de la escuela no es otro que ella misma en tanto que encarnación cumplida de lo modélico como universal (Fullat, 2002a, 372). Mientras compartamos la necesidad del elemento normativo en pedagogía, y de elucidar la hipótesis de la pedagogía postmoderna se trate, lo que habrá que pensar es qué tipo de norma, postmoderna, podrá proponer esa pedagogía.

En referencia a la normatividad pedagógica, se puede distinguir entre una normatividad referencial, en un nivel descriptivo, en relación al discurso en el que se dice algo sobre cómo son las cosas, y también de una caracterización más pragmática del discurso, en la que se trata de pretender hacer cosas con lo que se dice (Larrosa, 1990, 15), en este caso con un cierto énfasis prescriptivo y un proponer cómo hacerlas. Y aunque una teoría pedagógica podría entenderse como normativa en la doble condición de referencial y pragmática, de hecho es este último sentido el que suele identificarse como normativo, oponiéndolo a meramente descriptivo o explicativo (Trilla, 2005, 294). Se trata de un enfoque normativo de la pedagogía que históricamente ha ido de la mano con la concepción de que "la teoría de la educación es teoría no porque su objeto de conocimiento sea teórico, sino porque se sirve de teorías para la mejora de la práctica educativa" (Colom, 2002, 186). Así entendido, todo pensamiento pedagógico es siempre teleológico porque trata siempre de resolver problemas, sean del tipo que fueren, y la preocupación pedagógica fundamental es "saber para hacer". Hasta aquí, siguiendo lo expuesto, no aparece inconveniente alguno en que una pedagogía de corte postmoderno pueda ser normativa. Sólo que, obviamente, deberá serlo con arreglo al tipo de norma relativista que caracteriza el pensamiento postmoderno que ya hemos explicado. Es decir, lo que no puede ser es universalista, en el sentido de que esa categoría y aspiración "se refiere absolutamente a todos los casos, [...] se inserta en el orden metafísico y responde a la necesidad" (Fullat, 2002a, 19).

Concluiríamos, pues, por el momento, que no deberíamos referirnos a la pedagogía postmoderna como una mera descripción o constatación empírica de un relativismo pedagógico o una educación delicuescente, puesto que entonces sí

5. Esto coincidiría con el tipo de pedagogía que Trilla (2005) ha llamado "teórico-práctica", por distinción de la puramente "experiencial" y la "especulativa". Según esto el pedagogo especulativo sería el que lee al filósofo (en el caso de este artículo, a Rorty, Gadamer o Lyotard) sólo para conocerlo mejor y no para que le ayude a saber cómo educar mejor. Sin embargo, si queremos una práctica educativa consciente, y con educadores conscientes de para qué están educando, hay que pasar cronológicamente antes por el tipo de saber -llámesele si se quiere especulativo- que comprende primero lo que dice el filósofo sobre las cosas y lo humano, y se decide después por uno u otro tipo de práctica educativa (sin que ello implique, como veremos en las secciones cuarta y quinta, que la teoría sea ese lugar donde habita el conocimiento de cuál es la mejor práctica). 
que estaríamos ante un simple conjunto de enunciados teóricos sobre la realidad. Se trata, en cambio, de una pedagogía, con perspectiva global y voluntad de coherencia interna, donde la relatividad se propone como valor normativo. Sostenemos, por tanto, que hay que entender la pedagogía postmoderna en base a una diferencia radical de planteamiento respecto a las pedagogías modernas, que es lo que le da entidad propia. Por todo lo dicho, nos parece que hay razones para reconsiderar la validez de las dos negaciones citadas (no hay pedagogías postmodernas porque no puede haberlas). Desde este punto de vista, no sólo puede haberlas, sino que, además, haylas. Nos remitimos en este sentido a trabajos aparecidos en los últimos años -abundaremos más adelante en algunos de ellos- (entre otros, Vandenberg, 2009; Todd, 2009; McWilliam, 2008; Carr, 2006; Biesta, 2005; Van Goor, Heyting y Vreeke, 2004; Masschelein y Ricken, 2003; Colom, 2002; Masschelein, 1998; Parker, 1997), que ya empiezan a constituir una cierta tradición y que, aun por vías e intensidades diversas, en su conjunto no sólo constatan sino que conforman propositivamente una pedagogía postmoderna.

\section{ElEMENTOS Clave EN LA CONCRECIÓN DE UNA PEDAGOGÍA POSTMODERNA}

Como se puede ver en las páginas de esta misma revista, es un hecho constatado que la disparidad de supuestos en las propuestas pedagógicas actuales es grande. Por un lado -y aun desde perspectivas muy distintas-, hay cierto acuerdo en la necesaria emergencia de una teoría de la educación con un nuevo modelo de "yo valioso", enfocado hacia "la apertura de la disponibilidad y no para la cerrazón de lo seguro", superador de la anterior perspectiva moderna y lógico-racional basada en la capacidad de conocer la verdad y medir lo real (López Herrerías, 2010, 84, 88). En una versión quizá más radical: una pedagogía soportada en una "antropología narrativa, que consistiría en aprender a vivir en la provisionalidad y la ambigüedad" y que pondría en cuestión el "camino" que "el sistema cultural e institucional aprueba y propone" (Mèlich, 2008, 121). Por otro lado, sin embargo, se articulan propuestas para recuperar «una visión ética, sólida, omnicomprensiva de la realidad, una visión dura de la educación como proceso de cambio teleológico hacia unos valores u otros" (Gil, 2008, 30). O un conocimiento normativo que nos señale "las mejores normas de acción en orden a unos fines preestablecidos" (Naval, 2008, 90). Más aún, se insiste en restaurar «el prestigio de la razón como capaz de verdad", ya que "vivir sin verdad es la peor desgracia" y hay que devolver a la educación "la posibilidad de decir algo serio sobre qué es el hombre, cuál el sentido de la vida y cómo puede vivirse una vida lograda y plena" (Barrio, 2008, 90, 97). Pero, claro, si no se dispone de una unidad de sentido compartida, ¿cuál proponer más allá del preguntarse por la misma? Precisamente la problemática postmoderna radica en con qué clase de legitimidad se puede optar, a voluntad, por imponer o proponer universalmente uno u otro modelo. Pertenecemos a la historia y la "factibilidad" de ésta ya hace tiempo que la Historia -como disciplina y como reflexión sobre ella- hizo patente 
que se trataba de una quimera. La sucesión de acontecimientos humanos resultante y cuyos testigos somos nosotros no la hacemos a voluntad, aunque suceda a través de nuestras intenciones y propósitos (Koselleck, 1993, 266). Ser consciente de eso no significa que no debamos seguir pensando, proponiendo e incluso luchando por la consecución exitosa de una u otra pedagogía, pero quizá sí que debamos reconsiderar el hacerlo con visos de relato fundamentador que ambiciona salvar a la humanidad de alguna suerte de decadencia o derrumbe moral.

\subsection{Relativismos y educación}

La posición relativista que late en la pedagogía postmoderna coincide con el punto de vista hermenéutico que se opone a "lo perenne y lo universal, lo que permanece invariante, lo regular y lo objetivo" y valora "la contingencia y el azar, lo singular, la situación y el detalle. En una palabra: lo ambiguo" (Mèlich, 2008, 121). Desde un punto de vista pedagógico normativo, lo ambiguo es lo que depende del contexto, lo que para ser valorado deberá ser puesto en relación a la situación dada. De ahí que para pensar el relativismo en relación a la educación nos será de gran ayuda distinguirlo en distintos tipos y grados.

Algunos autores lo han clasificado en cuatro variables, a saber: "el campo de discusión, el objeto relativizado, la radicalidad con que se mantiene la tesis relativista, y el contexto al que se reduce la validez de los enunciados en cuestión" (Arenas, Muñoz y Perona, 1997, 9-14). Atendiendo a esta taxonomía -aunque las dos primeras variables las podríamos reunir en una, puesto que ambas tratan de aquello que se relativiza (Sánchez Durá, 1997, 146)-, el tipo de relativismo que habilita a una pedagogía postmoderna se inspira en lo siguiente: En cuanto al campo de discusión, sus enunciados toman forma de tesis epistémicas, éticas y estéticas, y encajan con una pedagogía que quiere formar para un mundo con verdades, valores y gustos relativos. Respecto al objeto, entre otros posibles -como la idea de educación, de educando, de educador, etc.- se podría articular un relativismo metodológico (que atendería especialmente a los medios que se dispondrían según se adecuaran a cada situación). En cuanto a la radicalidad, cualquier pedagogía que no quiera convertirse en mera improvisación debe desmarcarse del relativismo radical o irrestricto, puesto que supondría renunciar a todo postulado pedagógico. Es el caso de la pedagogía postmoderna, que se basa en un relativismo no ingenuo, es decir, que reconoce un límite a la validez de sus afirmaciones, pero que, dentro de ese ámbito en el que queda recortada la validez de su discurso, una afirmación verdadera o un valor compartido lo son de modo lógicamente absoluto. Y en referencia al contexto o a los factores relativizadores de los métodos, contenidos y valores de la educación, éstos podrían ser de muchos tipos. Por poner sólo algunos ejemplos: teorías del aprendizaje respecto a los métodos, la actualidad local respecto a los contenidos, la tradición cultural respecto a los valores, etc. 
Esta taxonomización del relativismo y su traducción pedagógica requerirán, claro está, un estudio y un desarrollo didáctico ulteriores. Sin embargo, ya algunas concreciones didácticas se han ensayado. Vandenberg (2009), por ejemplo, ha señalado dos modos de mantener la verdad en la enseñanza compatibles con el postmodernismo. Uno, afirmando que cada disciplina tiene su método y su modo de acercarse a lo real, no necesitando de ninguna metanarrativa de conocimiento absoluto que abarque todos los dominios o disciplinas para poder afirmar una verdad en un determinado campo. Dos, aceptando una combinación flexible de las principales teorías del conocimiento como detentoras cada una de medias verdades, y proponiendo esta combinación no como una síntesis sino como un "agregado flexible ${ }^{6}$. Ambos casos representarían, como se ve, una reconceptualización a la baja del predicado "verdadero", pero una reconceptualización que goza hoy de reconocidos argumentos filosóficos -como los de Quine-, desde los cuales puede haber "teorías diferentes y a la vez empíricamente adecuadas dentro de un mismo dominio", esto es, con postulados distintos pero con idéntico apoyo evidencial y similar justificación empírica, y ser ambas verdaderas (Muñoz, 1997, 27).

No hace mucho, en una sesión académica, alguien se preguntó si desde una pedagogía postmoderna estaría permitido matar al educando. Preguntar a qué tipo de límites morales se adscribe una pedagogía, o si suscribe la universalidad de los derechos humanos, es (a no ser que se pregunte retóricamente) una pregunta "fundacionalista" que encierra en el fondo la búsqueda de una respuesta unívoca basada en el esquema moderno de un metarrelato compartido del que no se pueda dudar. Quien escribe esto, como seguramente quienes lo están leyendo, esperan que ningún educador asesine a su educando, pero una pedagogía postmoderna no puede basarse en ningún fundamento absoluto desde el cual se permitan o prohíban, legitimen o no, metafísica ni metaéticamente ciertas acciones. Esa legitimidad y permisión deberá provenir de la reflexión crítico-racional que sobre sus acciones libres cada educador debe hacer y sobre la que, además, cada sociedad deberá tomar sus acuerdos. Aun así, quizá esto no dependa de si se trata de un planteo pedagógico moderno o postmoderno. Para el caso podríamos preguntarnos si desde una pedagogía moderna, por ejemplo, la makarenkiana, estaba legitimado sacudir a los educandos. Desde el planteamiento pedagógico no lo estaba, y, sin

6. Aunque no lo podemos desarrollar en este artículo, VANDENBERG propone una articulación didáctica de seis niveles en cuyo sexto estadio se pretende, a través de lo que llama la "flexibilidad epistémica", "desocultar algunas verdades parciales" por medio de focalizar y dirigir la atención de los estudiantes hacia un mismo "fenómeno en el mundo", para así ayudarlos a "explorar su mundo, y no el del profesor" (ibid., 163). Sólo entonces, como dice HEIDEGGER en el $₫ 44$ de Ser y Tiempo (2003, 246-250), cuando la verdad depende de que haya alguien para intuirla, ésta puede aparecer entendida como el desocultamiento de las cosas, mostrando así la "aperturidad" que es el modo de ser esencial de lo que ha sido desvelado. Aunque está por desarrollar, el trabajo de Vandenberg, así como el de otros fenomenólogos, abre vías para compatibilizar el método fenomenológico con los postulados relativistas postmodernos, atendiendo, por ejemplo, al concepto de Lebenswelt intersubjetivo. 
embargo, ocurría e incluso podía juzgarse como una acción contextualmente positiva ${ }^{7}$.

Un juicio moral, como el de que matar está mal siempre, puede ser concebido objetivamente, puesto que quien así lo defiende piensa que matar está mal independientemente de que eso sea lo que nuestra tradición piensa, lo que no puede es ser validado objetivamente. "Alguien que mantiene una concepción objetivista de sus juicios morales no niega que esté juzgando desde una tradición, lo que ocurre es que esto es irrelevante para él a la hora de mantener su actitud moral" (Espada, 1997, 231). Una cosa es la denuncia filosófica postmoderna de la tesis de la universalidad de tradiciones que son históricas y contingentes, por ejemplo, de una tradición moral. Otra muy distinta es afirmar que cualquier acción vale y que no hay posibilidad de convenir criterio alguno de conducta. Y aún otra es pedirle a una pedagogía que constituya una lista de mandatos imperativos en términos morales. Si toda moral es un conjunto de valores al que una voluntad libre puede o no adherirse, eso incluye también a una moral universalista que proponga un universalismo convenido o contingente y que podría concretarse de muy distintas formas. Todd ha propuesto participar de una pedagogía con un cierto tipo de universalidad normativa, arguyendo precisamente que la mayoría de críticos postmodernos lo que hacen son enmiendas parciales a los universalismos absolutos, pero no a la totalidad del concepto de universalidad. Desde ahí se podría sostener, como hace Gil $(2011,27)$, que "cabe perfectamente una aspiración teórica universalista, al tiempo que adaptada a las circunstancias, a los sujetos y a las culturas". Sin embargo, el tipo de universalismo que rechazan los postmodernistas es el que se basa en la lógica de la exclusión, y que descansa en la negación de lo concreto ya en su momento fundante (Todd, 2009, 20). Con lo que, de acuerdo con esa objeción, el universalismo postmoderno por el que se abogaría debería basarse en una normatividad líquida o muy susceptible de cambio, subordinando ésta a una "traducción cultural" constante, en función de cada contexto, y entendiendo esa labor como un proceso de adecuación y negociación cultural sin final, que no debe pretenderse como un momento intermedio para llegar a una meta ideal, sino como un fin en sí mismo (Todd, 2009, 21-22).

Considerar inaceptable la imposición de cualquier universalismo esencialista no significa que cada uno, desde el último filósofo hasta el primer educador, no pueda defender y luchar por aquella comunidad o tradición con la que se identifica en

7. «En honor a la verdad, yo no me sentía atormentado por ningún remordimiento de conciencia. Sí, había abofeteado a un educando. Yo experimentaba toda la incongruencia pedagógica, toda la ilegalidad jurídica de aquel hecho, pero, al mismo tiempo, comprendía que la pureza de mis manos pedagógicas era un asunto secundario en comparación con la tarea planteada ante mí. Estaba resueltamente decidido a ser dictador, si no salía adelante con ningún otro sistema. [...] Sin embargo, es preciso señalar que yo no pensaba ni por un minuto haber hallado en la violencia un medio todopoderoso de pedagogía". MAKARENKO (1977, 19-20). 
LA HIPÓTESIS DE LA PEDAGOGÍA POSTMODERNA. EDUCACIÓN, VERDAD Y RELATIVISMO

el presente. Como dijo Freire en la recepción de su doctorado bonoris causa en Madrid, "la posmodernidad significa no estar demasiado ciertos de nuestras certezas, sin que ello suponga descomprometerse. Renunciar a estar demasiado cierto de las certezas no significa negarlas, sino estar abierto a su superación y respetar las certezas de los otros" (citado en Novo, 1998, 98). Y es que no hay otro modo de trabajar por un mejor acuerdo de racionalidad o de moralidad que partir de la propia tradición (Putnam, citado en Rorty, 1991, 201). En síntesis, no existe contradicción en que un educador pueda ser consciente de su acto educativo, reconocerlo como deseable y defenderlo dentro de su tradición, y a su vez asumir la tesis relativista.

Lo postmoderno, dicho claro y raso, no significa que todo vale, sino que todo lo que vale depende. Partiendo de ahí, la pedagogía y la intencionalidad formativa son compatibles con el postulado postmoderno del relativismo tal como se ha definido. Incluso podría decirse que toda pedagogía que se inscriba dentro del marco hermenéutico y postmoderno tiene como principal pivote la idea de relatividad.

\subsection{Postfundacionalismo educativo}

Lo pedagógicamente más relevante del discurso postmoderno es su cuestionamiento de la jerarquización y sometimiento de cualquier práctica a un fundamento o a una metanarrativa apriorísticos. Por eso quienes presentan su mayor objeción a la Postmodernidad usando la acusación de relativismo como mayor argumento lo hacen en el sentido referido como radical o irrestricto. Pero eso es "intentar poner una metanarrativa en la boca del postmodernismo", identificando el "sostener una posición filosófica con el tener una metanarrativa disponible ${ }^{8}$.

A efectos de nuestra hipótesis, lo que más nos importa es que en nuestro presente postmoderno las metanarrativas fundacionalistas han caído en descrédito. El teórico de la educación Wilfred Carr afirmó que lo que hace "postmoderna" la teorización y la investigación educativas no es la disposición a renunciar al compromiso "moderno" con los valores emancipadores, sino la disposición a renunciar a la presunción de la Ilustración de que esos valores tengan que erigirse sobre unos "fundamentos" filosóficos" (Carr, 1995b, 164). También se ha advertido del peligro de las utopías propositivas y se ha resuelto que lo que la educación debería hacer no es tanto implementar o realizar proyectos y programas sino mantener permanentemente abiertos espacios y tiempos donde otros significados puedan ser pensados y articulados (Masschelein, 1998, 529-530). Incluso se ha llegado a afirmar que "los objetivos emancipadores de la Modernidad se han mostrado inútiles y hasta perniciosos. No existe un sentido único de la historia sino múltiples. El sujeto transformador de la sociedad tiene que desaparecer si queremos librarnos de los

8. Y añade Rorty: "Si insistimos en tal definición de "filosofía”, entonces el postmodernismo es post-filosófico. Pero sería mejor cambiar la definición” (RORTY, 1991, 202). 
dictadores. ¿Y la ciencia? Es una narrativa más, débil como cualquier otro relato o cuento. Hay una certeza universal, y es ésta: todo es relativo" (Fullat, 2002a, 371). Aunque resulte una aseveración un tanto provocativa, es claramente indicativa del sentir y pensar postmoderno. Un pedagogo podrá defender y luchar por promover cualesquiera proyectos educativos, pero

ya no procurará ofrecer garantías filosóficas de los tipos de teorización y de investigación que defiende, reconociendo sin lugar a dudas que no hay ningún punto de vista ahistórico desde el que patrocinar los valores emancipadores que trata de promover. Al conceder que no hay nada exterior a la experiencia, ni "esencia" de la naturaleza humana ni "destino" hacia el que inevitablemente se dirija la historia, también se concede que el único modo de justificarse consiste en apelar a su fe en la disposición de los profesionales corrientes de la educación a reconstruir su práctica de manera que exprese los valores e ideales educativos emancipadores (Carr, 1995a, 164).

Con todo, y como iba también a reconocer una década después el mismo Carr (2006), pese a "las tentativas por ver en este discurso de la posmodernidad la continuación de un enfoque crítico e incluso tecnológico, lo cierto es que el mismo supone una ruptura que sitúa la discusión sobre las posibilidades del conocimiento pedagógico en un plano radicalmente diferente" (Bárcena, 2005, 38)9. Tal y como dijo Parker en su Manifesto for education in postmodernity, para los educadores postmodernos una teoría general es precisamente algo que ya no puede ser escrito, con lo que no queda otra que remitirse a relatos sobre tal o cual situación y a pensar y discutir en cada lugar y momento los juicios y acciones que se puedan proponer. Cada decisión del educador no podrá estar fundamentada en una realidad ni en una justificación última (Parker, 1997, 142-144).

En los últimos años ya se ha referido al pensamiento educativo postmoderno en términos de postfundacionalismo, definido como el discurso filosófico actualmente vigente que

9. Aunque hay mucha literatura sobre la imposibilidad postmoderna del llamado enfoque crítico de la educación [véanse, por ejemplo, los artículos de G. BIESTA y J. MASSCHELEIN, entre otros, en el volumen 48 (4) de Educational Theory (1998), o algunos de los trabajos de GIROUX (1997) u otra vez BIESTA (2005) dedicados al tema], es importante resaltar que la solución habermasiana del "Consenso alcanzado de acuerdo a principios correctos de argumentación" ha sido considerada como de "fundacionalismo débil", dado que en ella el procedimiento de reglas de argumentación racional hacía las funciones de un principio primero, igual que los religiosos o políticos al uso (VAN GOOR, HEYTING y VREEKE, 2004, 176, 191). En los textos de Carr de los años noventa se pueden apreciar, todavía, elementos de ese enfoque. Por ejemplo, cuando propone: "una forma de razonamiento práctico que no supone traducir a la praxis principios teóricos, sino que requiere la deliberación colectiva sobre los atractivos relativos de diversos cursos alternativos de acción. [...] la forma de razonamiento que guía la acción práctica en una sociedad democrática, sociedad que ya no se funda en las certidumbres del conocimiento teórico y prefiere, en cambio, operar sobre la base de la creencia práctica contingente" (CARR, 1995a, 109-110). 
reconoce que los cambios irreversibles en las formas en las cuales ahora entendemos y nos relacionamos con las ideas y creencias de la modernidad han sido tan profundos que las formas de teorizar que continúan basándose en asunciones fundacionalistas ya no son aceptables si intentamos hacernos cargo del mundo contemporáneo (Carr, 2006, 150).

Según Carr, desde que existe la disciplina, todos los debates de la teoría de la educación sobre sus fundamentos epistemológicos (de la filosofía a la ciencia, de la colección de disciplinas académicas a las teorías propias de los educadores) han tenido lugar con arreglo a la autoevidente necesidad de ese proyecto teórico. Un proyecto basado en asumir como natural que toda actividad práctica como la educación está, o debería estar, basada en algún tipo de teoría, lo que a juicio del pedagogo británico es ya insostenible (ibid., 146). Si esto es así, ante una pedagogía postmoderna se podría seguir diciendo, incluso con más sentido, aquello de Ortega de: "el pedagogo no ha sido nunca el filósofo de su pedagogía". No porque se identifique -como advierte y desaconseja Rorty- "filosofía" con metanarrativa o verdad externa que guía la práctica, que también. Sino porque, como ya mantenía Carr (1995a, 111) refiriéndose a Democracia y Educación como una obra precursora del postmodernismo y que "pronostica una estrategia educativa "desfilosofizada" o "posfilosófica" ", los educadores postmodernos no se comprometen con teorías ni con actividades o articulaciones teóricas, sino sólo con las creencias que subyacen a sus prácticas (Carr, 2006, 154-155).

A nuestro modo de ver, esto no tiene por qué negar la intencionalidad educativa, ni pedagogías que se guíen por ideales u orientaciones, puesto que, como ha escrito Marchart $(2009,84)$ a propósito de la política, el problema no es la imposibilidad de cualquier fundamento, "sino la imposibilidad de su fundamento último, y es justamente esta ausencia radical, la condición de posibilidad de los múltiples, plurales y contingentes actos de "fundarla". En paralelo, esto tampoco niega la consideración, en un plano explicativo más alejado, de que los discursos pedagógicos dominantes en cada caso sean el marco de referencia a partir del cual se legitiman históricamente las concreciones políticas, las legislaciones o las prácticas educativas (Moreu, 2000, 149). Esos discursos son los que construyen la realidad pedagógica en tanto que entidad significativa. Tal que así, el discurso de la pedagogía postmoderna construye la idea y la imagen que los individuos (profesionales de la educación o no) tienen de la realidad pedagógica, y en virtud de la cual actúan. Una imagen que, al rechazar el modelo fundacionalista de justificación, asume, como decían Carr, Rorty, Gadamer, etc., la naturalidad de la falta de un fundamento teórico para la práctica. La pérdida del potencial prescriptivo y crítico del fundamento se concibe como una limitación que quiere tornarse en ventaja: "En vez de convertir la situación en otra forma de universalismo o validez general, tomar esa carencia como punto de partida" (Van Goor, Heyting y Vreeke, 2004, 186). 


\section{CONCLUSIONES}

Es cierto que la filosofía y la pedagogía postmodernas presentan inconsistencias si intentamos, con mentalidad y afán sistemáticos, poner en relación todas sus críticas y propuestas, pero también presentan un caldo común que está constituyendo un cambio de imaginario social y pedagógico que no podemos pasar por alto. No podemos dejar de tomar en serio, como ha dicho Rubio Carracedo (1996, 89), el nuevo ethos que esas críticas y propuestas encarnan en cuanto a nuevo talante moral y pedagógico. Pero también hay que hacer notar que a veces resulta demasiado fácil decir, ora como táctica evasiva ora como fórmula de cortesía, que la crítica postmoderna, aunque no se asuma en su totalidad, presenta muchas aportaciones positivas que hay que recoger. Desde antiguo suele darse esa situación cuando hay alguna propuesta realmente diferenciada y rupturista con lo anterior $^{10}$.

Por otra parte, tal vez el problema no sea tanto cómo llamar a esa pedagogía, ni discutir si es o no normativa, como aceptar su realidad y su necesidad contextual. Es decir, que, una vez y mientras permanezcamos dentro de la condición social postmoderna, no puede no ser. No se trata de que el estado de las cosas pueda resumirse en una postura maniquea según la cual antes todo era moderno y ahora todo es postmoderno. Tampoco es que -parafraseando a Chesterton- hablar de la Postmodernidad y el descrédito de las grandes narrativas a los jóvenes educadores, siempre y en todos los casos, sea anunciar la muerte de algo a quienes ni sabían que existía. Pero sí que en muchas facultades de formación de educadores comienza a ser bastante así. Quizá todos deberíamos contemplar la hipótesis de que las generaciones nacidas y crecidas al calor de la condición postmoderna no van a poder educar en las mismas coordenadas normativas desde las que lo han hecho las precedentes. Y si no van a poder es porque parten de un conjunto distinto de supuestos. Es posible que hoy se esté abriendo un hiato intergeneracional, no ya entre padres adultos e hijos adolescentes (Gervilla, 1993, 157), sino ahora ya entre abuelos, por un lado, y padres y nietos, por otro. En ese caso, podríamos decir hoy que entre los miembros de la comunidad educativa -incluyendo a la alta academia- coexisten los dos imaginarios sociales - moderno y postmoderno-. Y si éstos fueran inconmensurables, entonces no todos estaríamos siquiera en condiciones de hacernos cargo y aceptar la dimensión pedagógica del otro imaginario.

10. Mariano Carderera, por tomar un ejemplo de los inicios de la pedagogía, dice en la entrada "Jacotot" de su diccionario: "Mucho me he detenido en exponer los desvaríos de un pedagogo fuera de juicio. Sin embargo no se puede menos que reconocer que su método ha producido bien; pues ha hecho que se examinen nuevamente las combinaciones antiguas y promovido otras. Tal es la suerte que tienen comúnmente las innovaciones; fecundar el pensamiento y promover el bien por otros medios, ya que ellas no lo hacen por sí mismas. Examinadlo todo y conservad lo bueno, decía, como es sabido, el apóstol San Pablo" (CARDERERA, 1858). 
$\mathrm{Y}$ es que aunque algunos de nosotros podamos estar persuadidos de la necesidad de fundamentación y valores fuertes, sólidos y absolutos, sugiriendo que una educación postmoderna puede llevar a la destrucción de la persona y la sociedad (Gervilla, 2010, 235), esa concepción, nos guste o no, ha dejado de ser compartida. Tanto si nos identificamos como pedagogos postmodernos como si luchamos contra ellos o simplemente constatamos la existencia de éstos y esa suerte de pensamiento, no hacemos sino verificar la tesis postmoderna de la pluralidad y coexistencia de distintas verdades y metarrelatos. Como suele decirse, cuando hay un eclipse, todos estamos a oscuras.

¿Es posible compartir la necesidad normativa de la pedagogía (Trilla, 2005), pero también que "de nada sirve un proyecto filosófico que se refiera solamente a condiciones normalizadas de la existencia" (Bárcena, 2005, 12)? Si la respuesta es afirmativa, la pedagogía postmoderna podría inscribirse de algún modo en lo que se consideró la necesaria negación de la teoría educativa de la Modernidad para poder elaborar una nueva base teórica, ineludible si se comparte que el saber para hacer y la mejora de la práctica es lo que define toda teoría de la educación (Colom, 2002, 184-186). Ahora bien, otros pedagogos reputados - "modernos" en su momento- sostienen hoy que lo que se debería hacer es abandonar el proyecto de la teoría de la educación, asumiendo que ésta no puede tener "implicaciones prácticas" y que, por consiguiente, pierde el sentido que la animó durante el último siglo, que no era otro que el de arbitrar y evaluar, desde una posición neutral e independiente, la racionalidad, objetividad, validez y verdad de las creencias y prácticas educativas (Carr, 2006, 156).

Si la carencia de un sentido único y fuerte es una de las constantes postmodernas, tal vez se podría pensar en dar como tarea a los filósofos, y quizá también a los pedagogos, la recuperación o reconsideración del sentido, incluyendo la posibilidad de que ese sentido fuera, precisamente, la pluralidad de sentidos. No en vano se ha considerado que quizá «el sentido de la escuela pueda ganar en consistencia si somos capaces de considerar el conjunto de sus posibles sentidos" (García del Dujo y Gil, 2009, 100). Pero, bien mirado, ése no sería el signo de lo postmoderno, el cual se identificaría más bien con algo así como la disminución radical de las pretensiones de cualquier sentido ${ }^{11}$. Esto último es relevante porque señala precisamente lo que diferencia la normatividad postmoderna, postfundacionalista, de las pedagogías sostenidas en el fundacionalismo (especialmente del

11. Precisamente en la línea de Odo Marquard que ya García del Dujo y Gil Cantero citan y apuntan como alternativa: "... La experiencia moderna y actual de la pérdida de sentido es la consecuencia de un exceso de pretensiones en relación al sentido; así pues, nuestra dificultad primaria no es la pérdida de sentido, sino el exceso de pretensión de sentido; y lo que nos saca adelante no es el gran lamento por la pérdida de sentido, sino una moderación (una reducción) de la pretensión excesiva de sentido: una dieta de sentido mediante una dietética de la expectativa de sentido" (Marquard, citado en GARCÍA DEL DuJO y GIL, 2009, 100). 
llamado "fundacionalismo débil", que jerarquiza y sigue haciendo depender las prácticas de una justificación, aunque ésta sea relativa y contextual). Por eso, ante la recurrente y primitiva crítica que acusa al postmodernismo de negar el principio que propone (poner como fundamento el que no hay fundamento o pretender validez universal a la afirmación de que no hay validez universal), Van Goor, Heyting y Vreeke (2004, 190-191), después de haber estudiado numerosos trabajos postmodernos sobre educación, concluyen que el mero uso de procedimientos racionales para negar la validez universal no puede convertir a sus autores en fundacionalistas. Porque no se puede obviar, arguyen, "el distinto significado que los filósofos antifundacionalistas atribuyen a los procedimientos de justificación. En primer lugar, porque su validez es considerada contextual por naturaleza, y en consecuencia, ni la justificación científica o filosófica implican necesariamente la justificación de la práctica".

Quizá alumbren algo propuestas como las que afirman que "la teoría pedagógica debe ser la "teoría de lo único", es decir, del caso particular, [...] [que] abandona simbólicamente al niño, de un modo reflexivo, para estar con él de un modo real" (Van Manen, 2003, 165). Quizá precisamente a alumbrar la práctica, o a inspirarla, pero no a dirigirla, sea a lo único que hoy la teoría de la educación pueda aspirar. Y nos parece necesario distinguir entre una cosa y la otra porque, hoy en día, esa coletilla que afirma que toda práctica educativa debe tener en cuenta el contexto, que no puede pretenderse aplicar un esquema general para situaciones educativas siempre particulares, se ha convertido en un lugar común (como el de fomentar la crítica y la autonomía), al que los más dispares y opuestos planteamientos ideológicos dicen siempre adherirse (Masschelein, 2004, 351). Sin embargo, una cosa es querer comprender el fenómeno educativo en su contexto, y otra hacerlo "Con vistas a regular la acción educativa en ámbitos formales, no formales e informales", añadiendo que, "además de regular, cabría hablar de dirigir y conducir la acción educativa" (Naval, 2008, 57). Son cosas distintas, lo que hemos tratado de traer a colación en este artículo es la idea de una "nueva normatividad" que evita las prescripciones y las decisiones deducibles de una teoría, y cuya "emergente novedad radica en su orientación hacia la apertura y la indecidibilidad" (Van Goor, Heyting y Vreeke, 2004, 192). A nuestro modo de ver, esta nueva normatividad, postfundacionalista, sí puede estar de acuerdo con la necesidad de "más teoría" para profesores y educadores que defiende el profesor Gil, siempre que sea, como dice, para la "justificación reflexiva y especulativa sobre el sentido educativo del motivo adoptado" (Gil, 2011, 39), es decir, de las decisiones situadas del educador, y no como justificación general de una práctica.

Si hoy ya muchos pedagogos asumen que las decisiones, las prácticas y las creencias implícitas de las que éstas se derivan no son legítimamente generalizables ni evaluables desde un fundamento último, si incluso muchos teóricos de la educación -o quienes trabajan e investigan bajo el rótulo de esa área de conocimiento- asumen lo mismo o bien proceden con ese supuesto implícito sin cuestionárselo... ¿No será eso ya una pedagogía postmoderna? La pedagogía ya 
no puede hoy ser esa parte del saber práctico que, como en la división de saberes de Petzelt, sustentada en la historia y la ética, se ocupaba de "procurar que, en el futuro, [los actos libres del hombre] sean lo que deban ser" (Tusquets, 1972, 25). Para comprender y ahondar en los posicionamientos filosófico-pedagógicos de esa nueva pedagogía habrá que, a nuestro juicio, fijarse en sus ascendencias de corte hermenéutico y pragmatista. Se trata de filosofías en las que el relativismo no es sino la subordinación de la verdad a lo que resulta más educativo y formador: el sentido de mantener una conversación cuyo fin no es reflejar hechos con exactitud, sino resultar formativa dentro de las circunstancias de cada tradición.

Con todo, es cierto que el debate sobre si hay o no pedagogía postmoderna, que se haría inacabable en el continuo esgrimir unas u otras definiciones, quizá resulte en última instancia estéril para la pedagogía. "Por encima de la realidad está la posibilidad", decía el $₫ 7$ de Ser y tiempo (op. cit., 61). Es muy probable que nos encontremos ante un planteamiento pedagógico irreductible a cualquier otra cosa anterior, no ya cronológicamente, sino incluso desde un punto de vista lógico y conceptual. Por eso el mismo plantear la pedagogía postmoderna como hipótesis tal vez sólo pueda ser un cuestionamiento moderno, desde cuyos supuestos esa hipótesis funciona casi como una pregunta retórica que se responde negándola por principio ${ }^{12}$. En ese sentido, desde el pensamiento fundacionalista moderno, la pedagogía postmoderna sería casi un oxímoron. En cambio, desde el postfundacionalismo la misma pregunta tendría poco sentido práctico ${ }^{13}$. Así lo confesaba Wilfred Carr en la entrevista que le hicieron Jover y Thoilliez $(2010,60)$ refiriéndose a todas las preguntas que han buscado fundamentos teóricos y normativos para la práctica de la educación: "La razón por la que no hemos llegado a responderlas no es porque sea difícil, sino porque es imposible, porque son preguntas sin sentido".

John Dewey escribió (citado en Rorty, 2001, 41):

... persiste la convicción -aunque la historia muestra que es una alucinación- de que todas las preguntas que la mente humana ha planteado son preguntas que pueden ser respondidas en términos de las alternativas que las mismas preguntas ofrecen. Pero, de hecho, el progreso usualmente ocurre a través del

12. Se llega a esta conclusión al final del desarrollo de la investigación presentada en el artículo, por ello el título de este trabajo es el que es y mantiene el planteo abierto del inicio de la misma. Sin embargo, dadas las conclusiones a que se ha llegado, quizá ahora debería titularse "la hipótesis moderna de la pedagogía postmoderna", circunscribiendo histórica y paradigmáticamente ese enfoque, o bien, si se asumen las tesis postmodernistas, eliminando lo de "hipótesis" para reconocer abiertamente la existencia de la "pedagogía postmoderna".

13. Tendría poco sentido práctico, tanto si entendemos el término en su uso común de conjunto de meras destrezas técnicas aparentemente ateóricas como si lo hacemos, más rigurosamente, añadiendo que éstas forman parte de un marco teórico implícito, una tradición, sólo en virtud de la cual cobran sentido. En el primer significado, la pregunta, en tanto que teórica, no tendría vinculación con la práctica, en el segundo, la pregunta por el fundamento carecería de sentido práctico porque la tradición de la que hablamos se caracteriza por sostener la no necesidad de los fundamentos. 
craso abandono de las preguntas junto con las alternativas que presuponen, un abandono que resulta de su vitalidad decreciente y de un cambio en la urgencia del interés. No las resolvemos; pasamos sobre ellas.

Tal vez la pedagogía postmoderna como hipótesis, la pregunta que nos divide, la que duda sobre cómo volver a fundar una educación basada en el conocimiento y la verdad, sea a lo que aluden tanto el pragmatista como el hermeneuta. Hagamos lo que hagamos no podemos escapar del tiempo.

\section{REFERENCIAS BIBLIOGRÁFICAS}

AyUste, A. y TRILla, J. (2005) Pedagogías de la modernidad y discursos postmodernos sobre la educación. Revista de Educación, 336, 219-248.

BÁrcena, F. (2005) La experiencia reflexiva en educación. Barcelona, Paidós.

BARRIO, J. M. (2008) Educación y verdad. Teoría de la Educación. Revista Interuniversitaria, 20, 83-99.

BIESTA, G. (1998) Say you want a revolution... suggestions for the impossible future of critical pedagogy. Educational Theory, 48 (4), 499-510.

- (2005) What Can Critical Pedagogy Learn from Postmodernism? Further Reflections on the Impossible Future of Critical Pedagogy, en GuR ZE'Ev, I. (ed.) Critical Theory and Critical Pedagogy Today, Toward a New Critical Language in Education. Haifa, University of Haifa, 143-159.

CABrera, M. Á. (2001) Historia, lenguaje y teoría de la sociedad. Madrid, Cátedra.

- (2005) La crisis de la modernidad y la renovación de los estudios históricos, en FERRAz, M. (ed.) Repensar la bistoria de la educación. Madrid, Biblioteca Nueva, 21-52.

CARDERERA, M. (1858) Diccionario de la educación y métodos de enseñanza. Madrid, Imp. de A. Vicente.

CARR, D. (2005) El sentido de la educación. Barcelona, Graó.

CARR, W. (1995a) Educación y democracia: ante el desafío postmoderno, en AA.VV. Volver a pensar la educación. Política, sociedad y educación (vol. I). [Congreso Internacional de Didáctica celebrado en 1993]. Madrid, Morata.

- (1995b) Una teoría para la educación. Hacia una investigación educativa crítica. Madrid, Morata.

- (2006) Education without theory. British Journal of Educational Studies, 54 (2), 136-159.

Child, M.; Williams, D.; BIRCH, A. y BoODY, R. (1995) Autonomy or Heteronomy? Levinas's Challenge to Modernism and Postmodernism. Educational Theory, 45 (2), 167-189.

Colom, A. (1984) Hacia nuevos paradigmas educativos: la pedagogía de la postmodernidad. Studia Paedagogica, 14, 17-38.

- (2002) La (de)construcción del conocimiento pedagógico. Barcelona, Paidós.

ESPADA, C. (1997) El relativismo como cuestión ética, en ARENAS, L.; MUÑOZ, J. y PERONA, Á. (eds.) El desafio del relativismo. Madrid, Trotta, 227-240.

Foucault, M. (2003) Las palabras y las cosas. Buenos Aires, Siglo XXI.

Fullat, O. (2002a) Pedagogía existencialista y postmoderna. Madrid, Síntesis.

- (2002b) El siglo postmoderno (1900-2001). Barcelona, Crítica. 
LA HIPÓTESIS DE LA PEDAGOGÍA POSTMODERNA. EDUCACIÓN, VERDAD Y RELATIVISMO

- (2005) Valores y Narrativa. Axiología educativa de Occidente. Barcelona, Publicacions de la Universitat de Barcelona.

GADAMER, H.-G. (1994) Verdad y método II. Salamanca, Sígueme.

- (2003) Verdad y método I. Salamanca, Sígueme.

GARCía DEL DuJO, Á. y GIL, F. (2009) Reconstrucción del sentido de la actividad educativa escolar, en PEÑA, J. V. y FERnÁndeZ, C. (coords.) La escuela en crisis. Barcelona, Octaedro.

Gervilla, E. (1993) Postmodernidad y educación. Madrid, Dykinson.

- (2010) Educar en la postmodernidad. Madrid, Dykinson.

GIL, F. (2008) Ciudadanía y humanidad. La educación en el disenso. Teoría de la Educación. Revista Interuniversitaria, 20, 25-44.

- (2011) Educación con teoría. Revisión pedagógica de las relaciones entre la teoría y la práctica educativa. Teoría de la Educación. Revista Interuniversitaria, 23 (1), 19-43.

Giroux, H. (1997) Border Pedagogy in the Age of Postmodernism, en GIROUX, H. (ed.) Pedagogy and the Politics of Hope: Theory, Culture, and Schooling. Boulder, Westview, 147-163.

Hansen, K. (2008) Rewriting Bildung for Postmodernity: Books on Educational Philosophy, Classroom Practice, and Reflective Teaching. Curriculum Inquiry, 38, 93-115.

Heidegger, M. (2003) Ser y Tiempo. Madrid, Trotta.

Husserl, E. (1987) La filosofía como ciencia estricta. Buenos Aires, Nova.

JOVER, G. y ThOYlliez, B. (2010) Cuatro décadas de teoría de la educación: ¿una ecuación imposible? Teoría de la Educación. Revista Interuniversitaria, 22 (1), 43-64.

Koselleck, R. (1993) Sobre la disponibilidad de la historia, en Futuro Pasado. Barcelona, Paidós.

LARrosa, J. (1990) El trabajo epistemológico en pedagogía. Barcelona, PPu.

LAUDO, X. (2008) L'educació i la societat del coneixement. Els reptes d'un món globalitzat, en GONZÁlEZ, J. Una societat en crisi? Reptes d'un món globalitzat. Vic, Eumo, 191-211.

- (2011) El ideal de formación del Tao y su pedagogía líquida, en VERGARA, J.; SÁNCHEZ, F. y COMELLA, B. Ideales de formación en la historia de la educación. Madrid, Dykinson, 23-37.

LÓPEZ HERRERÍAs, J. A. (2010) ¿Qué "yo" es valioso para el mundo de hoy? Teoría de la Educación. Revista Interuniversitaria, 22 (1), 65-90.

LYON, D. (1994) Postmodernidad: la historia de una idea, en Postmodernidad. Madrid, Alianza.

LYOTARD, J. F. (1994) La condición postmoderna. Madrid, Cátedra.

LYOTARD, J. F. y DESCAMPS, C. (1984) Jean-François Lyotard dans la sociètè post-moderne, en Entretiens avec "Le Monde", vol. 1, Philosophes. París, La Dècouverte/Le Monde, 1984, 149-157.

MAILLARD, Ch. (1998) La razón estética. Barcelona: Laertes.

Makarenko, A. S. (1977) Poema pedagógico. Barcelona, Planeta, 19-20.

Marchart, O. (2009) El pensamiento político posfundacional. Buenos Aires, Fondo de Cultura Económica.

Masschelein, J. (1998) How to Imagine Something Exterior to the System: Critical Education as Problematization. Educational Theory, 48 (4), 521-530.

- (2004) How to Conceive of Critical Educational Theory Today? Journal of Philosophy of Education, 38, 351-367. 
MAsschelein, J. y RicKen, N. (2003) Do We (Still) Need the Concept of Bildung? Educational Philosophy and Theory, 35, 139-154.

MCWILliam, E. (2008) Unlearning how to teach. Innovations in Education and Teaching International, 45 (3), 263-269.

MÈLICH, J.-C. (2008) Antropología narrativa y educación. Teoría de la Educación. Revista Interuniversitaria, 20, 101-124.

MichÉA, J.-C. (2002) La escuela de la ignorancia y sus condiciones modernas. Madrid, Ediciones Acuarela.

Moreu, Á. (2000) Pedagogia política i política educativa. Temps d'Educació, 24, 141-168.

MUÑOZ, J. (1997) La pluralidad de los mundos (Notas sobre realismo y relativismo), en ARENAS, L.; Muñoz, J. y Perona, Á. (eds.) El desafío del relativismo. Madrid, Trotta.

Naval, C. (2008) Teoría de la Educación. Pamplona, EUNSA.

NieTZSCHE, F. (1999) Así habló Zaratustra. Madrid, Alianza.

NiÑO, L. (2008) Modernidad y narrativa posmoderna. Estética, 9, 191-202.

Novo, M. (1998) Pedagogía y posmodernidad: Ni olvido de la historia ni relativismo moral. Cuadernos de Pedagogía, 265, 86-89.

Nubiola, J. (2001) Pragmatismos y relativismo. C. S. Pierce y R. Rorty. Themata. Revista de Filosofía, 27, 49-57.

PANIKKAR, R. (2007) Mito, fe y hermenéutica. Barcelona, Herder.

PARKER, S. (1997) Reflective teaching in the postmodern world. A manifesto for education in postmodernity. Buckingham, Open University Press.

Quintana PaZ, M. Á. (2008) Pequeña reflexión de traza wittgensteiniana en torno a hermenéutica y educación, en EsTEBAN ORTEGA, J. (ed.) Cultura, hermenéutica y educación. Valladolid, UEMC/CEINCE.

ROMI, S. y SCHMIDA, M. (2009) Non-formal education: a major educational force in the postmodern era. Cambridge Journal of Education, 39: 2, 257-273.

RORTY, R. (1991) Objectivism, Relativism, and Truth. Philosophical papers, volume 1. Cambridge, Cambridge University Press.

- (1995) La filosofía y el espejo de la naturaleza. Madrid, Cátedra.

- (2001) ¿Esperanza o conocimiento? Una introducción al pragmatismo. México, Fondo de Cultura Económica.

RUBio CARRACEDO, J. (1996) Educación moral, postmodernidad y democracia. Madrid, Trotta.

TAYLOR, C. (2002) Modern social imaginaries. Public Culture, 14 (1), 91-124.

Terrén, E. (1997) Postmodernidad y educación. Politica y sociedad, 24, 121-140.

TODD, S. (2009) Universality and the Daunting Task of Cultural Translation. Journal of Philosophy of Education, 43 (1), 1-29.

Trilla, J. (2005) Hacer pedagogía hoy, en Ruiz Berrio, J. (ed.) Pedagogía y educación ante el siglo XXI. Madrid, Universidad Complutense de Madrid, 287-309.

TusqueTs, J. (1928) Assaigs de crítica filosòfica. Barcelona, Edicions de la Nova Revista.

- (1972) Teoría de la Educación. Madrid, Magisterio español.

VAn Goor, R.; Heyting, F. y VReeke, G.-J. (2004) Beyond Fundations: Signs of a New Normativity in Philosophy of Education. Educational Theory, 54 (2), 173-192.

VAN MANEN, M. (2003) Investigación educativa y experiencia vivida. Barcelona, Idea Books.

VANDENBERG, D. (2009) Critical thinking about truth in teaching: The epistemic ethos. Educational Philosophy and Theory, 41 (2), 155-165.

Zubiri, J. (1926) Filosofía del ejemplo. Revista de Pedagogía. Madrid, 55, 289-285. 